9) Schuerman L, et al. Vaccine 2009; 27: 5748-54.

10) Novotny L, et al. Vaccine 2006; 24: 4804-11

4

\section{THE EVOLVING ADOLESCENT IMMUNIZATION SCHEDULE: A NEED FOR ACELLULAR PERTUSSIS BOOSTER IMMUNIZATIONS}

\section{W. Joel}

UCLA Center for Vaccine Research, Torrance, Torrance, CA, USA

Routine use of pertussis vaccines globally has led to dramatic declines in the incidence of whooping cough in young children. Yet pertussis is incompletely controlled and causes about 300,000 deaths per year worldwide. ${ }^{1}$ Most pertussis cases now occur in the unimmunized, young infants prior to completing their initial pertussis immunizations and importantly in adolescents and adults with waning immunity. In developed countries, infections are now transmitted predominately from older individuals (reservoir of infection) to younger infants who suffer greater morbidity and mortality.

There are several acellular pertussis vaccines for children (variations of DTaP) and lower dose booster formulations for adolescents and adults (Tdap). These vaccines are effective, generally well-tolerated and have clinically acceptable safety profiles, especially in comparison to the whole cell vaccines. However, protective immunity to pertussis wanes over $5-10$ years after immunization. ${ }^{2}$

In addition to preventing pertussis, tetanus and diphtheria, there are multiple reasons for instituting a routine adolescent immunization visit between 10-15 years of age. It also provides an opportunity to immunize with meningococcal and HPV vaccines and for selective use of vaccines such as HBV, $M M R$, varicella, influenza and hopefully in the near future, vaccines such as herpes type II. Importantly it is also an opportunity to reach adolescents for health screening, education and counseling.

This presentation will review the formulation, immunogenicity, and safety of aP vaccines as well as the rationale for pertussis boosters in the current epidemiological context. The ultimate control of pertussis will require routine pertussis booster immunizations for adolescents and adults as well as for the parents of young infants (cocoon strategy).

References:
1) WHO. D.o.v.a.B.W.H. Organisation. Geneva. 2003. Available from: http://www.who.int/vaccinesdocuments/DocsPDF06/843.pdf [accessed April 2010]. 2) Hellenbrand W, et al. BMC Infect Dis 2009; 9: 22.

\section{5}

\section{SUCCESSES AND REMAINING CHALLENGES IN MENINGOCOCCAL DISEASE PREVENTION}

\author{
J. Vázquez \\ National Institute of Health Carlos III, \\ Madrid, Spain
}

Neisseria meningitidis is a leading cause of invasive meningococcal disease (IMD) with a high casefatality rate, causing 50,000 deaths worldwide annually. ${ }^{1}$ Five serogroups of $N$. meningitidis (A, $B, C, W-135$ and $Y$ ) cause the majority of human disease. ${ }^{2}$ Prevalence of each serogroup varies temporally and geographically, ${ }^{2,3}$ and new strains can spread rapidly around the world. Protection against IMD can be achieved using monovalent ( $A$ and $C$ only) and multivalent (A, C, W-135 and $\mathrm{Y})$ polysaccharide (PS) meningococcal vaccines, which have demonstrated efficacy and a clinically acceptable safety profiles in children $>2$-years-old. ${ }^{4}$ However, in infants $<2$ years, these vaccines are poorlyimmunogenicand donotinduceimmunological memory. Furthermore, duration of immunity is short (3-5 years) and hyporesponsiveness can occur after repeated doses. ${ }^{4}$ Effective protein-polysaccharideconjugate vaccines are licensed for use in children $<2$-years-old, ${ }^{5}$ but only provide protection against serogroup C.GlaxoSmithKline is developing a tetanus toxoid-conjugated tetravalent serogroup A, C, W-135, Y vaccine (MenACWY-TT), which potentially could address some of these issues. In clinical trials, the candidate vaccine demonstrated similar immunogenicity against serogroup C (seroresponse rates $>94.8 \%$ ) to MenC-conjugated vaccines in children aged $1-10$ years.$^{6-9}$ Additionally, immunogenicity of MenACWY-TT post-vaccination was non-inferior to MenACWY-PS vaccines against all four serogroups in adolescents/adults aged $11-55,{ }^{10,11}$ with significantly higher MenA, MenW135 and MenY geometric mean antibody titres 1 year post-vaccination in 11-17-year-olds. ${ }^{11}$ In clinical trials, MenACWY-TT has shown a clinically acceptable reactogenicity and safety profile. ${ }^{12,13}$

\section{References:}

1) WHO. Wkly Epidemiol Rec 2002; 77: 329-40. 